Proceedings of the International Symposium on Physics of Materials (ISPMA 14), September 10-15, 2017, Prague

\title{
Deformation Behavior of Mg-alloy-based Composites at Different Temperatures Studied by Neutron Diffraction
}

\author{
G. FARKAS ${ }^{a, b, *}$, J. Pilch ${ }^{b}$, P. MinÁRIK ${ }^{a}$ AND K. MÁThiS ${ }^{a}$ \\ ${ }^{a}$ Department of Metal Physics, Charles University, Ke Karlovu, 5, CZ-121 16 Prague, Czech Republic \\ ${ }^{b}$ Nuclear Physics Institute, v. v. i., 25068 Rež, Czech Republic
}

\begin{abstract}
Deformation mechanisms in a $\mathrm{Mg}-\mathrm{Al}-\mathrm{Ca}$ alloy reinforced with short alumina fibres were studied by acoustic emission and neutron diffraction method (ND). The microstructure after deformation was observed by electron microscope (SEM). The fibres plane orientation with respect to the loading axis was found to be a key parameter, which influences the active deformation processes, such as twinning and dislocation slip. In-situ neutron diffraction tests were performed at room temperature $(\mathrm{RT})$ and $100^{\circ} \mathrm{C}$. The relative twin volume in the matrix as a function of the applied compressive load were evaluated from the ND tests. Acoustic emission results indicate a larger twinning activity and more frequent fibre cracking in sample with perpendicular fibre plane orientation. Both of these mechanisms increase in magnitude at $100^{\circ} \mathrm{C}$.
\end{abstract}

DOI: 10.12693/APhysPolA.134.881

PACS/topics: 61.72.Mm, *43.40.Le, 61.05.fm

\section{Introduction}

Magnesium alloys are the lightest metallic structural materials. Therefore they have been attracting substantial attention in engineering for the last several decades. $\mathrm{Mg}$ alloys have been widely used particularly in aerospace and automotive industry due to their high specific strength, low density and excellent damping properties. Nevertheless, some of their properties as e.g. low creep resistance, low ductility or degradation of the mechanical properties at elevated temperatures limit their application potential. These disadvantages can be considerably eliminated by adding of ceramic reinforcement phases, as e.g. $\mathrm{SiC}$ or $\mathrm{Al}_{2} \mathrm{O}_{3}$ to the matrix alloy [1-4].

In composites besides the deformation mechanisms operating in the matrix (basal and non-basal dislocation slip, twinning) [5] reinforcement effects as e.g. enhanced dislocation density, load transfer from the matrix to fibers or grain refinement [4] are present. Thus, the mechanical properties of the composite are influenced by the volume fraction of the fibers, as well as by theirs orientation with respect to the loading axis.

The acoustic emission (AE) and neutron diffraction (ND) are a powerful and reliable experimental techniques for investigating deformation processes in composites. Both of them gain experimental data from a large volume of the specimen. The AE is an elastic wave arising from sudden energy release due to local dynamic changes in the microstructure caused by the internal or external forces. Consequently, the AE technique is capable to detect acoustic signals originating from the processes connected with plastic deformation of composites such as collective dislocation motion, deformation twinning or fiber cracking [6].

*corresponding author; e-mail: farkasgr@gmail.com
Neutron diffraction method was used for the investigation of the activity of twinning during deformation [7]. Owing to the large penetration depth of the thermal neutrons, this technique is able to bring information about the deformation processes characterizing the whole gauge volume. Since the diffraction pattern is sensitive on the texture variation, the relative twin volume can be measured from the intensity changes of particular, twin related grains [8].

The present work focuses on the investigation of the twinning and dislocation activity during deformation (under an applied stress) in the unreinforced AX41 magnesium alloy and AX41 alloy reinforced with short Saffil ${ }^{\circledR}$ fibers. The influence of the fibers' orientation with respect to the loading axis is discussed in details.

\section{Experimental}

AX41 magnesium alloy with a nominal composition of Mg-6 wt.\%Al-1 wt.\% Ca was reinforced with 25 vol.\% short Saffils fibers $\left(\delta-\mathrm{Al}_{2} \mathrm{O}_{3}\right)$. The material was prepared by squeeze casting technique. The preform, consisting of $\mathrm{Al}_{2} \mathrm{O}_{3}$ short fibers showing a 2D planar isotropic fiber distribution and a binder system (containing $\mathrm{Al}_{2} \mathrm{O}_{3}$ and starch) were preheated to $1000^{\circ} \mathrm{C}$, inserted into a preheated die $\left(300-360^{\circ} \mathrm{C}\right)$ and then infiltrated by the liquid alloy using two-stage application of the pressure. The mean fibers length and fibers diameter (measured after squeeze casting) were $\sim 78 \mu \mathrm{m}$ and $\sim 3 \mu \mathrm{m}$, respectively. Specimens for deformation tests were cut from the bulk with two orientations with respect to the fibers plane: (i) with the fibers plane parallel $(\mathrm{PaO})$ and (ii) perpendicular to the longest sample axis which is identical with the loading direction $(\mathrm{PeO})$. Samples were not thermally treated. The specimens having dimension $20 \times 10 \times 10 \mathrm{~mm}^{3}$ were deformed in compression at room temperature and $100^{\circ} \mathrm{C}$ with an initial strain rate 
of $10^{-3} \mathrm{~s}^{-1}$. In addition, samples made from the matrix alloy samples without any reinforcement were also investigated.

The AE response was detected by a computercontrolled PCI-2 system manufactured by Physical Acoustic Corporation (PAC). The facility incorporated a Micro2006 transducer (fabricated by DAKEL-ZD Rpety) with a flat response between 50 and $650 \mathrm{kHz}$ and a PAC $2 / 4 / 6$ preamplifier giving a gain of $40 \mathrm{~dB}$. The threshold level was set at $26 \mathrm{~dB}$, directly above the peak values of the background noise.

Neutron diffraction measurements were performed in a biaxial diffractometer TKSN-400 optimized for the study of internal stresses in polycrystalline materials. Diffracted neutrons exhibited the wavelength of $\lambda=2.3 \AA$, the neutron flux was approximately $10^{5} \mathrm{n}$ per $\mathrm{cm}^{2} / \mathrm{s}$. The detector position was fixed during the whole experiment. Duration of each measurement was $4 \mathrm{~h}$. The gauge volume was situated inside the sample with two $5.5 \times 5.5 \mathrm{~mm}^{2}$ slits.

\section{Results}

\subsection{Microstructure}

The microstructure of the AX41 composite sample after deformation is shown in Fig. 1. Fibres have clearly random orientation in fiber planes (Fig 1a-d). In case of $\mathrm{PeO}$ the scanned plane (Fig. 1a, c) is the cross section of the specimen while in $\mathrm{PaO}$ the observed plane is parallel with the loading axis (Fig. $1 \mathrm{~b}, \mathrm{~d}$ ). In the $\mathrm{PaO}$ specimens the occurrence of cracked fibers is low at room temperature (Fig. 1b). However, the probability of fiber failure increases at $100^{\circ} \mathrm{C}$ (Fig. 1d). For sample with $\mathrm{PeO}$ the fiber cracking is quite frequent even at RT (Fig. 1a).
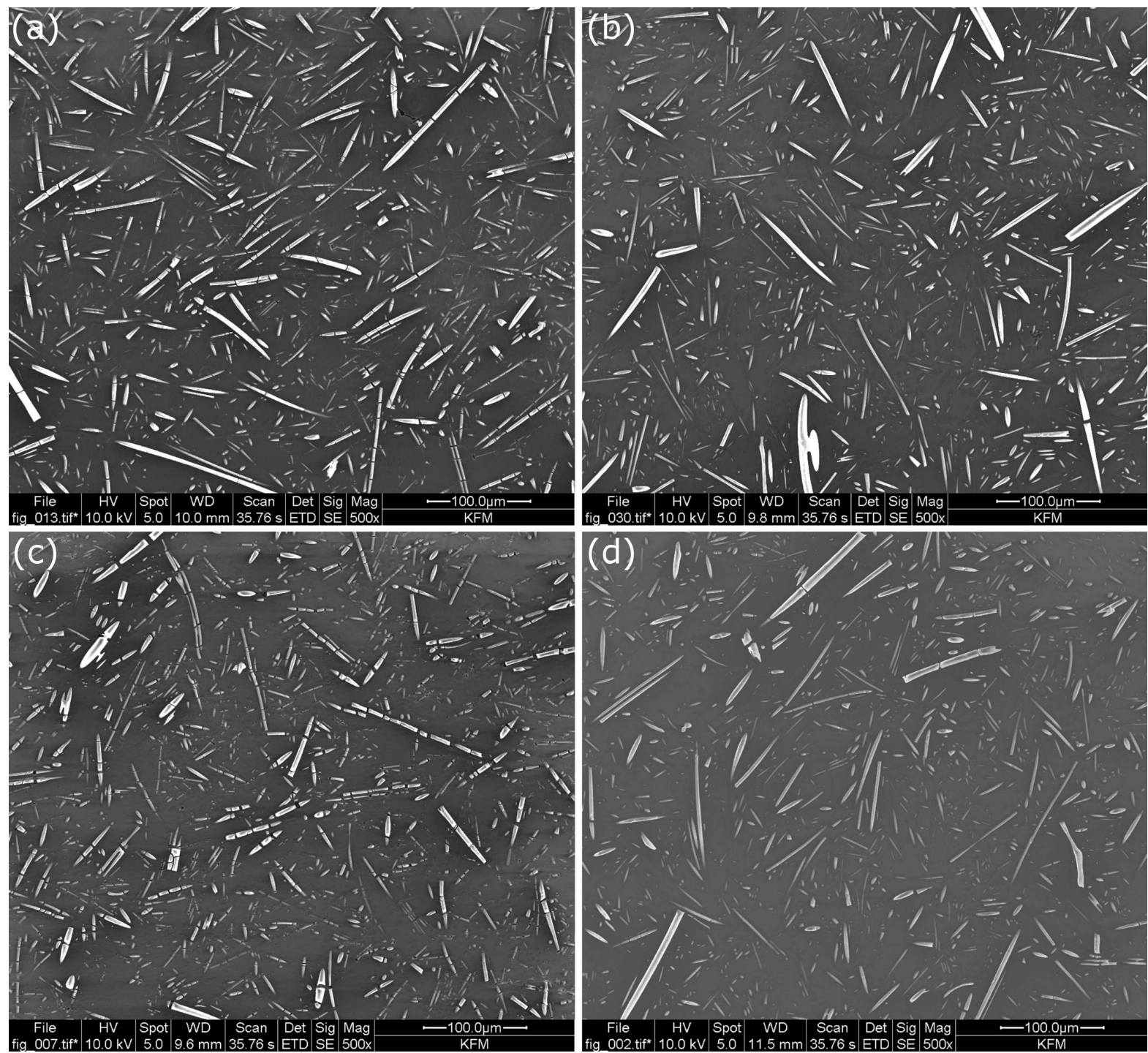

Fig. 1. Secondary electron images of the deformed specimen with (a) Perpendicular orientation $(P e O)(b)$ Parallel orientation $(\mathrm{PaO})$ at $\mathrm{RT}$ and with (c) $\mathrm{PeO}$ (d) $\mathrm{PaO}$ at $100^{\circ} \mathrm{C}$. 


\subsection{Acoustic emission}

The true stress-true strain curve for the alloy (Fig. 2a) and the composites with $\mathrm{PaO}$ (Fig. 2b) and $\mathrm{PeO}$ (Fig. 2c) deformed in compression at room temperature are depicted in Fig. 2. The yield stress and ultimate stress is the highest for $\mathrm{PaO}$ orientation $\left(\sigma_{y}^{P a O}=390 \mathrm{MPa}, \sigma_{y}^{P e O}=230 \mathrm{MPa}, \sigma_{m}^{P a O}=600 \mathrm{MPa}\right.$, $\sigma_{m}^{P e O}=410 \mathrm{MPa}$ ) which indicates an effective load transfer from matrix to reinforcement phase [9].
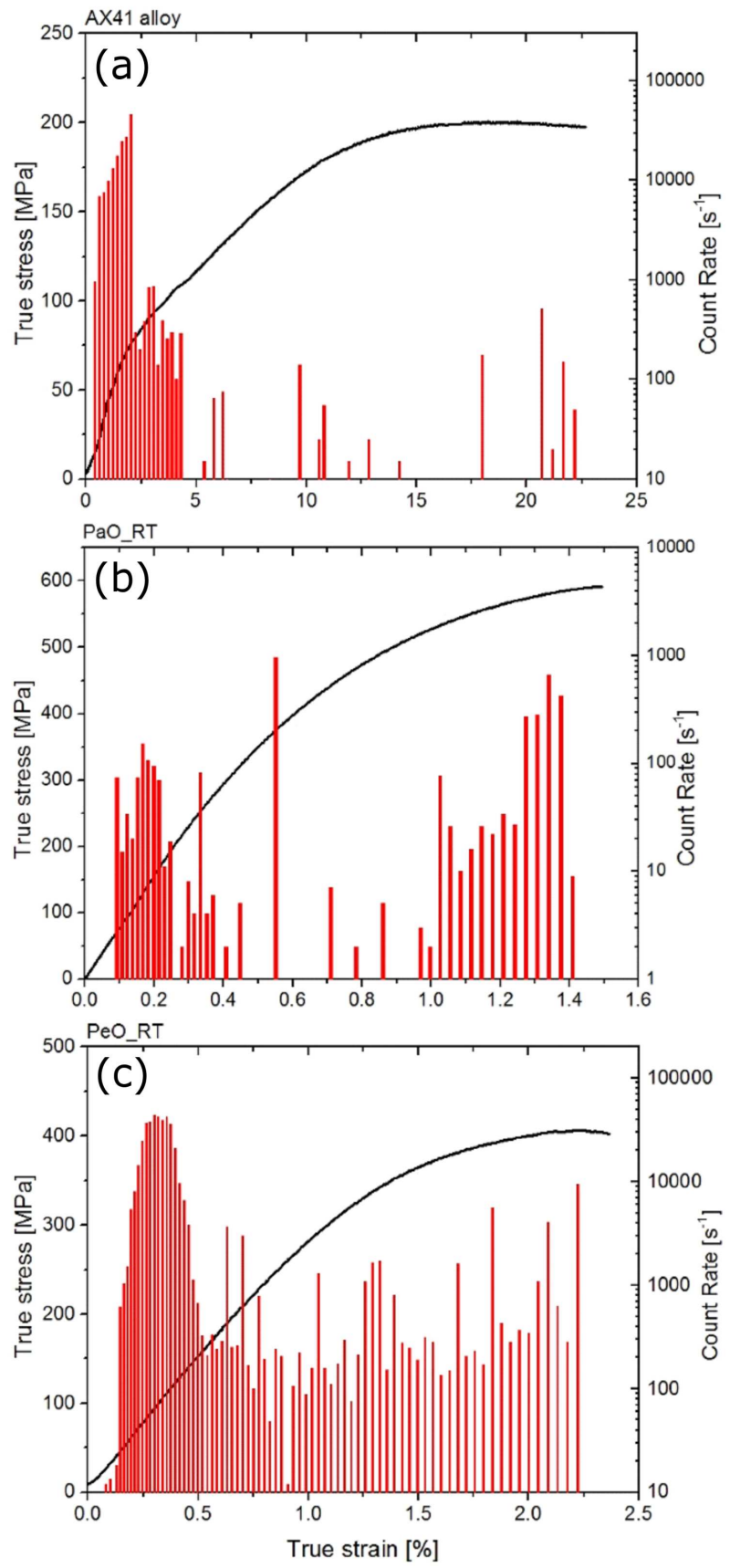

Fig. 2. True stress - true strain curves for (a) unreinforced alloy, (b) composite with $\mathrm{PaO}$, (c) with $\mathrm{PeO}$ deformed at room temperature.
The common feature of all AE curves is a significant peak at the onset of straining. This behavior can be attributed to the concurrent activity of dislocation slip in basal and prismatic planes and deformation twinning [10-12]. The steep decrease of the AE signal, which is typical for magnesium alloys, has two main reasons:

- The dislocation density increases with the increasing strain. Consequently, the mean free path of dislocations decreases. Since this parameter is directly proportional to AE intensity [13], a rapid drop of the AE signal is obvious.

- The twin nucleation can be expected as a significant AE source, since it is a result of rapid, collective motion of several hundred dislocations. As it is shown in our recent paper [14], in compression the twin nucleation is dominant only at the onset of straining. Above the macroscopic yield point the twin growth dominates, which is not detectable by $\mathrm{AE}$ [15]. Consequently, the AE signal at higher strains decreases under the detectable limit.

If we perform a closer inspection $\mathrm{AE}$ count rate for the particular samples, we can find several substantial differences between the AE response of composites $\mathrm{PaO}$, $\mathrm{PeO}$ and the unreinforced alloy, respectively:

- Lower AE activity in composites - this effect is caused mainly by the presence of the fibers, which act as obstacles for dislocation motion, and reduce the mean free path of dislocations. Further, the twin nucleation is suppressed owing to high internal stresses [16]. It is noteworthy that there is a higher intensity of the AE signal in the early stage of deformation for perpendicular fibers orientation. The $\{10 \underline{12}\}\langle 10 \underline{11}\rangle$-type extension twins are expected to be the main source of AE. In case of composite with $\mathrm{PaO}$ either the number of nucleated twins is lower or the size of twins, which also can be the reason for the lower AE intensity. The combination of more intensive twinning and a longer mean free path of dislocations could result in a higher intensity of $\mathrm{AE}$ in the $\mathrm{PeO}$ case.

- The AE signals in the terminal stage of the deformation in composites - the observed increase of the AE activity at higher strains is related to the fibers failure [16]. As it is obvious from Fig. 2b, c, this effect is more intensive for the sample with the perpendicular fibers plane orientation. This feature can be elucidated as follows: the force acting to fibers aligned with the loading axis is create compressive force on them. In contrast, forces which acting to fibers laying in the plane perpendicular to the loading axis bend the fibers. Such process can more easily break the fibers making an effective source of AE.

There are also similar features in the AE response of composites and matrix, respectively. The yield point correlates with the maximum of $\mathrm{AE}$ count rate. In case of 
unreinforced alloy the intensity starts to increase at low stresses $(\sim 40 \mathrm{MPa})$, which indicates that the twinning is actives at low stresses [14]. The twinning in the composite sample with $\mathrm{PeO}$ was initialized around $\sim 80 \mathrm{MPa}$, while the sample with $\mathrm{PaO}$ the activation starts approximately at $\sim 110 \mathrm{MPa}$ of applied stress. The onset of extension twinning before reaching the macroscopic yield point is evident and often reported also by other authors [17, 18].

\subsection{Acoustic emission at elevated temperature}

The true stress-true strain curves obtained in compression at $100^{\circ} \mathrm{C}$ are shown in Fig. 3 for both orientation of the fibers plane. The yield stress and ultimate stress is lower for both orientations $\left(\sigma_{y}^{\mathrm{PaO}}=270 \mathrm{MPa}\right.$, $\left.\sigma_{y}^{P e O}=190 \mathrm{MPa}, \sigma_{m}^{\mathrm{PaO}}=405 \mathrm{MPa}, \sigma_{m}^{P e O}=245 \mathrm{MPa}\right)$, than that for RT. The AE response is clearly higher at elevated temperature than at RT. This difference of AE activity mechanical properties is caused by the influence of thermally activated processes. It is believed that the non-basal slip systems (pyramidal and prismatic) is in background of this behavior [19]. Both twinning and the non-basal slip become easier at higher temperatures. The critical resolve shear stress (CRSS) at room temperature is almost 100 times higher than for basal slip. Since the Burgers vector of this systems is large in comparison to the lattice spacing of magnesium, the glide of these dislocations is rather difficult, requiring thermal activation. Consequently, the CRSS for the prismatic and pyramidal slip systems decreases rapidly with increasing temperature $[19,20]$.

It was measured previously with in-situ ND that large tensile strain is present in the matrix, which suppresses the twinning. The tensile strain reduces at elevated temperature making smaller influence on twinning [9]. Thus, twinning becomes more dominant and this process increases the activity of AE.

The AE signals in the terminal stage of the deformation in composites also increased, which indicates to the more frequent fiber cracking process and nucleation of cavities. The measured count rate suggests that fiber cracking in case of $\mathrm{PeO}$ is a more dominant mechanism than for $\mathrm{PaO}$.

\subsection{Twinning in $\mathrm{Mg}$ composites}

The twinning mechanism has significant effect on the diffraction peaks. Integrated intensity of particular reflections changes when twinning occurs. Therefore, ND experiments are excellent tools for estimation of the twinned volume. In compression, grains having their $c$-axis perpendicular to the loading axis, are optimally oriented for extension twinning $\{1012\}$ [13]. In axial direction, these grains have $\{10 \underline{10}\}$ and $\{11 \underline{2} 0\}$ orientations. The $\{10 \underline{12}\}$ extension twinning reorientates the grain by almost $90^{\circ}$ making parent-daughter relations between grains with reflections $\{10 \underline{10}\}-(0002)$ and $\{11 \underline{2} 0\}-\{10 \underline{1} 3\}$. Relative intensity decrease of the parent grain peaks is proportional to the twin volume. Concurrently, the relative intensity of the daughter grain orien- tations increases because the part of the newly emerging twins starts to contribute to the intensity of these reflections.
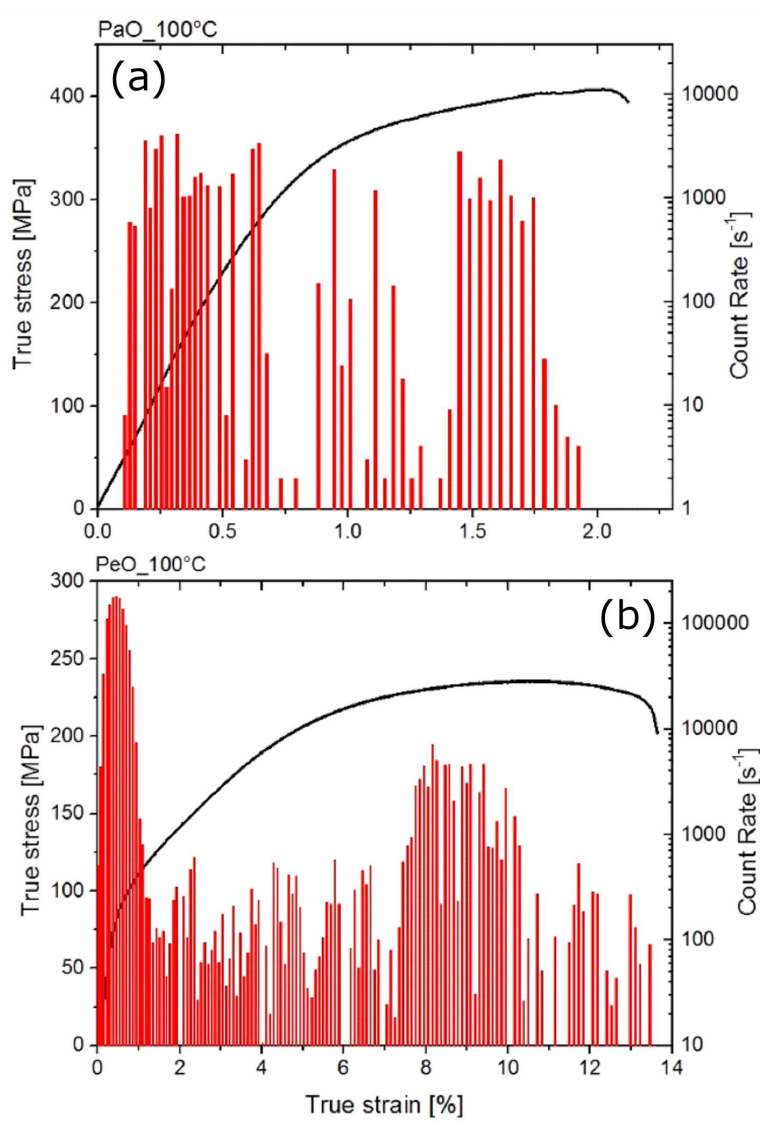

Fig. 3. True stress - true strain curves and work hardening rate curve with $\mathrm{AE}$ response for (a) composite with $\mathrm{PaO}$ (b) with $\mathrm{PeO}$ deformed at $100^{\circ} \mathrm{C}$.

In Fig. 4a-f the relative intensity changes of parent grains determined by single peak fits with respect to their initial state are presented. In axial direction the $\{1010\}$ and $\{1120\}$ reflections are shown for both $\mathrm{PaO}$ (Fig. 4c) and $\mathrm{PeO}$ (Fig. 4e) sample and also for the unreinforced alloy (Fig. 4a). In radial direction (0002) and $\{10 \underline{1} 3\}$ plane are in parent orientations for specimens (Fig. 4b) for $\mathrm{PaO}$ (Fig. 4d) and $\mathrm{PeO}$ (Fig. 4f). According to the results twinning activates much earlier for $\mathrm{PeO}$ than for $P a O$. The decrease of relative intensity takes place at $\sim 100 \mathrm{MPa})$. For samples with $\mathrm{PaO}$ twinning is virtually inactive up to $200 \mathrm{MPa}$. This behavior is different in case of magnesium alloys were the twinning is observed already at low applied stresses $(<10 \mathrm{MPa})$. This difference can be explained with the presence of fibers. The delayed activation of twinning on macroscopic scale in sample with $\mathrm{PaO}$ can be explained by the high LS in the matrix in axial direction [9]. The applied compressive load has to first compensate the tensile LS. 

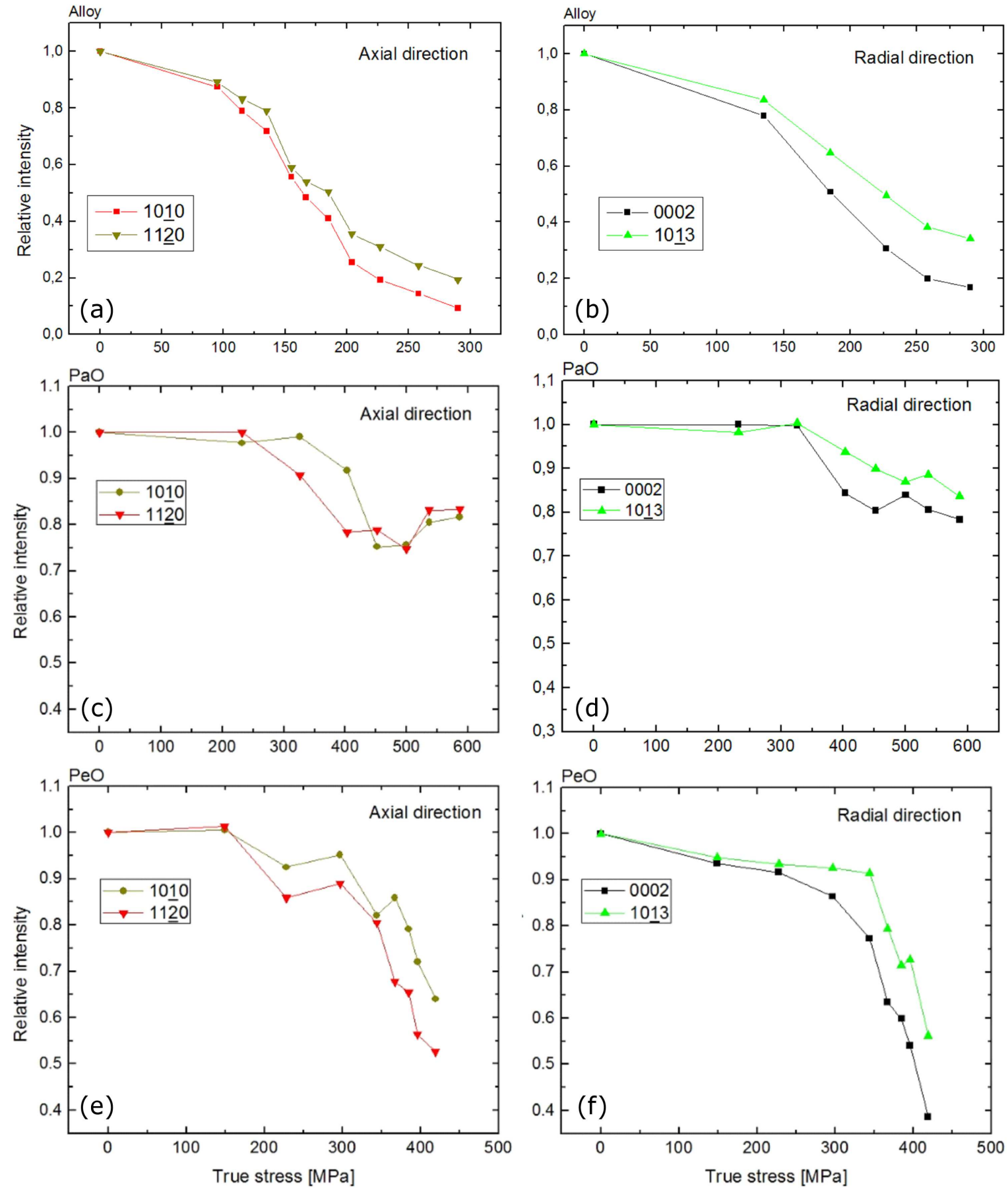

Fig. 4. Relative intensity evolution of the grains with "soft-orientation" for: (a, b) the alloy, (c, d) sample with $P a O$, $(\mathrm{e}, \mathrm{f})$ sample with $\mathrm{PeO}$. In axial direction (a, c, e) $\{1010\}$ and $\{1120\}$ peaks represent the parent grains while in radial direction $(b, d, f)$ the same role corresponds to (0002) and $\{10 \underline{1} 3\}$ reflections.

Only after this step the matrix is virtually compressed, which induces twinning. Another interesting difference between two orientations is the relative twin volume at the end of deformation. In sample with $\mathrm{PaO}$ twinning is less dominant, and approximately 20 vol.\% of favorably oriented grains undergo twinning. For $\mathrm{PeO}$ this ratio is around 60 vol.\% while for alloy it reaches the 80 vol.\%. This explains the larger ductility of $\mathrm{PeO}$ sample.

\section{Conclusions}

In this work AX41 composite reinforced with short Saffil fibers was investigated by both acoustic emission and ex-situ neutron diffraction methods. The influence of deformation temperature and fiber plane orientation on the deformation mechanisms was revealed. The measurements was completed with microstructure investigation by scanning electron microscope. 
The following conclusions can be drawn:

- Deformation tests show that parallel fiber plane orientation $(\mathrm{PaO})$ has larger reinforcing than the perpendicular one $(\mathrm{PeO})$.

- Twinning is more active for perpendicular fiber orientation $(\mathrm{PeO})$ and it activation starts at lower applied stresses than that for parallel orientation $(\mathrm{PaO})$. The relative twin volume is smaller than that for the alloy but much larger compared to the parallel orientation.

- The increasing testing temperature results in more frequent twin nucleation at the beginning of deformation for both type of fiber plane orientations.

- Fiber cracking is influenced by fiber plane orientation - in case of perpendicular orientation the probability of fiber cracking during deformation is larger. Fiber cracking is more frequent at higher temperature.

\section{Acknowledgments}

This work was financially supported by the Czech Science Foundation GACR under the Grant 14-36566G. Measurements were carried out at the CANAM infrastructure of the NPI CAS Rez supported through MŠMT project No. LM2015056. Presented results were obtained with the use of infrastructure Reactors LVR-15 and LR-0, which is financially supported by the Ministry of Education, Youth and Sports - project LM2015074.

\section{References}

[1] G. Farkas, K. Máthis, J. Pilch, Acta Phys. Pol. A, 128, 758 (2015).

[2] K.B. Nie, X.J. Wang, X.S. Hu, L. Xu, K. Wu M.Y. Zheng, Mater. Sci. Eng. A,528, 5278 (2011).
[3] Z. Trojanova, V. Gartnerova, A. Jäger, A. Námešný, M. Chalupová, P. Palček, P. Lukáč, Compos. Sci. Technol. 69, 2256 (2009).

[4] R. Mises, Math. Mech. 8, 161 (1928).

[5] G. Garces, G. Bruno, A. Wanner, Scr. Mater. 55, 163 (2006).

[6] Z. Trojanová, Z. Száraz, F. Chmelík, P. Lukáč, Mater. Sci. Eng. A,528, 2479 (2011).

[7] M.R. Daymond, C. Lund, M.A.M. Bourke, Metall. Mater. Tran. A 30, 2989 (1999).

[8] G. Farkas, Z. Trojanová, Z. Szaraz, P. Minarik, K. Máthis, Mater. Sci. Eng. A, 643, 25 (2015).

[9] G. Farkas, K. Máthis, J. Pilch, P. Minárik, P. Lukáš, A. Vinogradov, Mater. Sci. Eng. A 685, 284 (2017).

[10] M.R. Barnett, Mater. Sci. Eng. A 464, 1 (2007).

[11] A. Jain, S.R. Agnew, Mater. Sci. Eng. A 462, 29 (2007).

[12] K. Máthis, J. Čapek, Z. Zdražilová, Z. Trojanová, Mater. Sci. Eng. A 528, 5904 (2011).

[13] K. Máthis, F. Chmelík, in: Acoustic Emission, Ed. W. Sikorski, Intech Open Access Publisher, Rijeka 2012 , p. 23

[14] J. Čapek, K. Máthis, B. Clausen, J. Stráská, P. Beran, P. Lukáš, Mater. Sci. Eng. A 602, 25 (2014).

[15] A. Vinogradov, K. Máthis, JOM 68, 3057 (2016).

[16] K. Máthis, G. Farkas, M. Janeček, H. Choe, Mater. Sci. Eng. A 575, 1 (2013).

[17] Y. Li, M. Enoki, Mater. Sci. Eng. A 536, 8 (2012).

[18] A. Vinogradov, D. Orlov, A. Danyuk, Y. Estrin, Acta Mater. 61, 2044 (2013).

[19] K. Máthis, F. Chmelík, M. Janeček, B. Hadzima, Z. Trojanová, P. Lukáč, Acta Mater. 54, 5361 (2006).

[20] H. Yoshinaga, T. Obara, S. Morozumi, Mater. Sci. Eng. 12, 255 (1973). 